\title{
An Arithmetical-like Theory of Hereditarily Finite Sets
}

\author{
Márcia R. Cerioli ${ }^{1}$, Vitor Krauss ${ }^{2}$, Petrucio Viana ${ }^{3}$ \\ ${ }^{1}$ Instituto de Matemática \\ Programa de Engenharia de Sistemas e Computação - COPPE \\ Universidade Federal do Rio de Janeiro (UFRJ) - Rio de Janeiro - RJ - Brazil \\ ${ }^{2}$ Instituto de Matemática \\ Universidade Federal do Rio de Janeiro (UFRJ) - Rio de Janeiro - RJ - Brazil \\ Bolsista PIBIC/CNPq \\ ${ }^{3}$ Instituto de Matemática e Estatística \\ Universidade Federal Fluminense (UFF) - Niterói - RJ - Brazil \\ marcia@cos.ufr.br, kraussvitor@gmail.com, petrucio_viana@id.uff.br
}

\begin{abstract}
This paper presents the (second-order) theory of hereditarily finite sets according to the usual pattern adopted in the presentation of the (secondorder) theory of natural numbers. To this purpose, we consider three primitive concepts, together with four axioms, which are analogous to the usual Peano axioms. From them, we prove a homomorphism theorem, its converse, categoricity, and a kind of (semantical) completeness.
\end{abstract}

\section{Introduction}

Hereditarily finite sets (HFS) were defined by W. Ackerman in [Ackermann 1937] as the sets that satisfy all the usual first-oder Zermelo-Fraenkel axioms except the infinity axiom. Different first-order axiomatizations of the HFS theory were given by S. Givant and A. Tarski [Givant and Tarski 1977], P. Cohen [Cohen 2008], S. Swierczkowski [Świerczkowski 2003], and L. Kirby [Kirby 2009]. Alternatively, M. Takahashi [Takahashi 1976] and F. Previale [Previale 1994] developed the HFS theory using intuitionistic first-order logic, whereas Smolka and Stark [Smolka and Stark 2016] used intuitionistic type theory.

In ZF set theory, the natural numbers can be defined as the sets which can be obtained from $\emptyset$ by a finite number of applications of the successor function $s(x)=x \cup\{x\}$. Similarly, in ZF, the hereditarily finite sets can be viewed as a generalization of natural numbers, obtained by replacing the unary mapping $s(x)=$ $x \cup\{x\}$ by the binary mapping $S(x, y)=x \cup\{y\}$, and taking all the sets which can be obtained from $\emptyset$ by a finite number of applications of the new $S$. This similarity led us to construct the HFS theory as a generalization of the second order theory of natural numbers, defined by R. Dedekind in [Dedekind 1888] and G. Peano in [Peano 1889]. We adapt results from [Dedekind 1888, Givant and Tarski 1977, Lawvere 1964], and [Lawvere and McLarty 2005].

\section{The Peano axiomatization of the natural numbers}

In this section, we highlight the results that interest us about the axiomatization of the sequence of natural numbers, essentially due to Peano [Peano 1889]. We adopt concepts 
and notations which were not available at the time of the works of Dedekind and Peano. In particular, we use a second order language containing a constant symbol 0 and 1-place function symbol $s$.

A Peano structure, or simply P-structure, is an ordered triple $\mathcal{N}=\left\langle N, 0^{N}, s^{N}\right\rangle$, where $N$ is a non-empty set, $0^{N} \in N$, and $s^{N}: N \rightarrow N$. The domain of a P-structure $\mathcal{N}=\left\langle N, 0^{N}, s^{N}\right\rangle$ is $N$. We denote arbitrary P-structures by $\mathcal{N}, \mathcal{M}$ and their respective domains by $N, M$.

The Peano axioms, or simply $P$-axioms, are:

$$
\begin{array}{ll}
\mathrm{P}_{1} & \forall x[s(x) \neq 0] \\
\mathrm{P}_{2} & \forall x \forall y[s(x)=s(y) \rightarrow x=y] \\
\mathrm{P}_{3} & \forall X\{\langle X(0) \wedge \forall x[X(x) \rightarrow X(s(x))]\rangle \rightarrow \forall x[X(x)]\}
\end{array}
$$

where $x, y, z, \ldots$ are object variables ranging on the domains of the P-structures and $X, Y, Z, \ldots$ are set variables ranging on the set of subsets of the domains of the $\mathrm{P}$ structures.

We say that a $\mathrm{P}$-structure $\mathcal{N}$ is a Peano model, or simply a P-model, if all the $\mathrm{P}$ axioms are true in $\mathcal{N}$. The existence of a P-model $\left\langle N, 0^{N}, s^{N}\right\rangle$ can be proved in ZermeloFraenkel Set Theory by considering $N$ as the set of all sets which can be obtained from $0^{N}=\emptyset$ by a finite number of applications of $s^{N}(x)=x \cup\{x\}$. These are called the natural numbers sets.

One of the main consequences of the P-axioms is Theorem 1 below, which is called the Homomorphism Theorem. Recall that given P-structures $\mathcal{N}=\left\langle N, 0^{N}, s^{N}\right\rangle$ and $\mathcal{M}=\left\langle M, 0^{M}, s^{M}\right\rangle$, a homomorphism from $\mathcal{N}$ to $\mathcal{M}$ is a function $\Phi: N \rightarrow M$, such that $\Phi\left(0^{N}\right)=0^{M}$ and $\Phi\left(s^{N}(x)\right)=s^{M}(\Phi(x))$, for every $x \in N$.

Theorem 1 If $\mathcal{N}=\left\langle N, 0^{N}, S^{N}\right\rangle$ is a P-model, then for any P-structure $\mathcal{M}=$ $\left\langle M, 0^{M}, s^{M}\right\rangle$, there exists a unique homomorphism from $\mathcal{N}$ into $\mathcal{M}$.

PROOF. Essentially, in [Dedekind 1888].

The Homomorphism Theorem has as corollary the Categoricity Theorem. This is a fundamental result whose scope is the categoricity-i.e., the existence of a unique model up to isomorphism-of the P-axioms.

Theorem 2 If $\mathcal{N}=\left\langle N, 0^{N}, s^{N}\right\rangle$ and $\mathcal{M}=\left\langle M, 0^{M}, s^{M}\right\rangle$ are both P-models, then there exists a unique isomorphism from $\mathcal{N}$ onto $\mathcal{M}$.

ProOF. Essentially, in [Dedekind 1888].

The Categoricity Theorem implies further a sort of (semantical) completeness of the P-axioms.

Theorem 3 Let $\varphi$ be any statement in the (second order) language of the P-axioms. Then $\varphi$ is true on all P-models iff $\varphi$ is true on the natural numbers sets.

Proof. Essentially, in [Dedekind 1888]. 
An enlightening discussion on the real meaning of the Completeness Theorem 3, emphasizing the prerequisites involved in its formulation, its consequences and generalizations, is presented in [Awodey and Reck 2002].

Surprisingly-at least for these authors-it took a while for the converse of the Homomorphism Theorem to be investigated and proved. As far as we have been able to determine, the following beautiful result appears for the first time in [Lawvere 1964]albeit in a different context from that discussed here:

Theorem 4 If $\mathcal{N}=\left\langle N, 0^{N}, S^{N}\right\rangle$ is a P-structure and for any P-structure $\mathcal{M}=$ $\left\langle M, 0^{M}, s^{M}\right\rangle$, there exists a unique homomorphism from $\mathcal{N}$ into $\mathcal{M}$, then $\mathcal{N}$ is a P-model.

ProOF. See [Lawvere 1964, Lawvere and McLarty 2005].

The Homomorphism Theorem and its converse- that is, the whole of Theorems 1 and 4- were taken by Lawvere in [Lawvere 1964] as a categorical definition of natural number in any category having enough structure. Recall that a category $\mathbf{C}$ consists of a class of objects and a class of arrows satisfying a series of axioms. And, that, in this context, a category has enough structure if it is a topos, that is, it is Cartesian closed and has a sub-object classifier. An accurate presentation of these axioms, concepts and results may be found in [Lawvere and McLarty 2005].

\section{A Peano like axiomatization of the hereditarily finite sets}

In this section, we present a (second-order language) axiomatization of the hereditarily finite sets (HFS). Our axiomatization takes into account that the hereditarily finite sets can be viewed as a generalization of the natural number sets inside ZF set theory. Hence, our objectives are (1) to provide a set of axioms for HFS which have some resemblance with the P-axioms for the sequence of natural numbers; (2) to provide a proper notion of homomorphism in order to prove for the HFS results analogous to the homomorphism, categoricity, completeness and reverse of HT theorems. To this purpose, we define the HFS-axioms, the HFS-structures, the HFS-models, a proper notion of homomorphism and prove all these results. In particular, we use a second order language containing a constant symbol 0 and 2-place function symbol $S$.

A hereditarily finite sets structure, or simply HFS-structure, is an ordered triple $\mathcal{H}=\left\langle H, 0^{H}, S^{H}\right\rangle$, where $H$ is a non-empty set, $0^{H} \in H$, and $S^{H}: H \times H \rightarrow H$. The domain of a HFS-structure $\mathcal{H}=\left\langle H, 0^{H}, S^{H}\right\rangle$ is $H$. We denote arbitrary HFS-structures by $\mathcal{H}, \mathcal{G}$ and their respective domains by $H, G$.

The hereditarily finite sets axioms, or simply HFS-axioms, are:

$$
\begin{array}{ll}
\text { Zer } & \forall x[S(0, x) \neq 0] \\
\text { Ext } & \forall x \forall y\{\forall z[S(x, z)=x \leftrightarrow S(y, z)=y] \rightarrow x=y\} \\
\text { Ind } & \forall X\{\langle X(0) \wedge \forall x \forall y[X(x) \wedge X(y) \rightarrow X(S(x, y))]\rangle \rightarrow \forall x[X(x)]\} \\
\text { Red } & \forall x \forall y \forall z\{S(S(x, y), z)=S(x, y) \leftrightarrow[S(x, z)=x \vee z=y]\}
\end{array}
$$

where $x, y, z, \ldots$ are object variables ranging on the domains of the HFS-structures and $X, Y, Z, \ldots$ are set variables ranging on the set of subsets of the domains of the HFSstructures. 
We say that a HFS-structure $\mathcal{H}$ is a hereditarily finite set model, or simply a HFSmodel, if all the HFS-axioms are true in $\mathcal{H}$. The existence of a HFS-model can be proved in Zermelo-Fraenkel Set Theory by considering $0^{H}=\emptyset, S(x, y)=x \cup\{y\}$, and taking $H$ as the set of all sets which can be obtained from $0^{H}$ by a finite number of applications of $S$. These are called the hereditarily finite sets.

These HFS axioms are the second order correspondents of the first-order axioms proposed in [Givant and Tarski 1977]. Taking into account that intended meanings of 0 and $S(x, y)$, we can access their meanings. First, observe that under this interpretation we have that $S(x, y)=x$ iff $y \in x$. Whence:

- Zer, the zero axiom, is analogous to $\mathrm{P}_{1}$ asserting that the operation of including an element into the empty set results in a non-empty set.

- Ext, the extensionality axiom, is analogous to $\mathrm{P}_{2}$ asserting that sets which have exactly the same elements are equal. In fact, since $s(x)$ is a set, $P_{2}$ can be written as $\forall x \forall y\{\forall z[z \in s(x) \leftrightarrow z \in s(y)] \rightarrow x=y\}$.

- Ind, the induction axion, is analogous to $\mathrm{P}_{3}$ asserting that every subset that contains 0 as an element and is closed under $S$ contains all possible elements. In fact, since $S$ is binary, for a set $X$ to be closed under $S$, we need that $S(x, y) \in X$ for all $x, y \in X$.

- Red, the redundancy axiom, which has no analogous in Peano's axiomatization, asserts that a set belongs to a union $x \cup\{y\}$ iff it is already an element of $x$ or it is equal to $y$.

In a more extensive version of this article, that is currently in preparation, besides the results presented here, we prove that from these axioms follow all the basic constructions and results necessary for the development of a theory of the hereditarily finite sets. Besides, we investigate the relationship between this secondorder axiomatization and the second-order versions of the axiomatizations proposed in [Cohen 2008, Świerczkowski 2003, Kirby 2009, Takahashi 1976, Previale 1994], and [Smolka and Stark 2016]. Moreover, we prove two other principles of induction, both of which are consequences of Axiom Ind, and shall be employed in some of the proofs to come. The first of these principles, which we call a weak principle of induction, is stated as $\forall X\{X(0) \wedge \forall x\langle X(x) \rightarrow \forall y[X(S(x, y))]\rangle \rightarrow \forall x[X(x)]\}$. The second of these principles, which we call a strong principle of induction, is stated as $\forall X\{X(0) \wedge \forall x\langle\forall y[y \in x \rightarrow X(y)] \rightarrow X(x)\rangle \rightarrow \forall x[X(x)]\}$.

\section{Homomorphism, categoricity, and completeness for the hereditarily finite sets}

Our first main result is a version of the Homomorphism Theorem (cf. Theorem 1) for the hereditary finite sets.

Theorem 5 Let $\mathcal{H}=\left\langle H, 0^{H}, S^{H}\right\rangle$ be an HFS-model and $\mathcal{G}=\left\langle G, 0^{G}, S^{G}\right\rangle$ be an HFSstructure such that Axioms Zer, Ext and Red are true in $\mathcal{G}$. Then, there exists a unique function $\Phi$ such that:

(1) $\operatorname{Dom}(\Phi)=H$ and $\operatorname{Ran}(\Phi) \subseteq G$;

(2) $\Phi\left(0^{H}\right)=0^{G}$;

(3) For all $x, y \in H, \Phi\left(S^{H}(x, y)\right)=S^{G}(\Phi(x), \Phi(y))$; 
(4) $\Phi$ is injective.

PROOF. We begin by proving the existence of a least-w.r.t inclusion-relation $\Phi$ such that:

(1') $\Phi \subseteq H \times G$;

(2') $\left(0^{H}, 0^{G}\right) \in \Phi$;

(3') For all $x, y \in H$ and $z_{1}, z_{2} \in \mathcal{G}$, if $\left(x, z_{1}\right),\left(y, z_{2}\right) \in \Phi$, then $\left(S^{H}(x, y), S^{G}\left(z_{1}, z_{2}\right)\right) \in \Phi$

(4') For every $x \in H$ there exists $z \in G$ such that $(x, z) \in \Phi$, and (i) for every $v \in z$ there exists $u \in x$ such that $(u, v) \in \Phi$, and (ii) and for every $u \in x$ there exists $v \in z$ such that $(u, v) \in \Phi$.

First, note that $H \times G$ satisfies (1'), (2'), (3'), and (4'). Hence, $\mathcal{F}=\{X \subseteq H \times G$ :

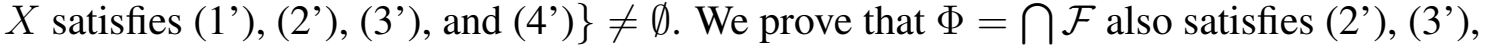
and (4'). The proofs of (2') and (3') are straightforward, so we omit them. To prove $\left(4^{\prime}\right)$, we proceed by weak induction on $x \in H$.

Basis: For $x=0^{H}$, take $z=0^{G}$. By Axiom Zer, $\forall u \in H[u \notin x]$ and $\forall v \in G[v \notin z]$. Thus $x$ and $z$ vacuously satisfy (i) and (ii).

Induction Hypothesis: Suppose that $\left(4^{\prime}\right)$ is true for $x$. That is, for $x$, suppose there exists $z_{1} \in G$ such that $\left(x, z_{1}\right) \in \Phi$ and $\left(x, z_{1}\right)$ satisfies (i) and (ii).

Induction Step: Suppose $\left(y, z_{2}\right) \in \Phi$. For $S^{H}(x, y)$, we take $S^{G}\left(z_{1}, z_{2}\right)$. Since $\left(x, z_{1}\right),\left(y, z_{2}\right) \in \Phi$, by $\left(3^{\prime}\right)$, we have $\left(S^{H}(x, y), S^{G}\left(z_{1}, z_{2}\right)\right) \in \Phi$. First we prove that $\left(S^{H}(x, y), S^{G}\left(z_{1}, z_{2}\right)\right)$ satisfies (i). Note that, by Axiom Red, $v \in S^{G}\left(z_{1}, z_{2}\right)$ if and only if $v \in z_{1}$ or $v=z_{2}$. We consider two cases. Case 1.1. If $v \in z_{1}$, by the $\mathrm{IH}$, there exists $u \in x$ such that $(u, v) \in \Phi$. Besides, by Axiom Red, $u \in S^{H}(x, y)$. Case 1.2. If $v=z_{2}$, there is $u=y$ such that $(u, v) \in \Phi$. Besides, by Axiom Red, $u \in S^{H}(x, y)$. Now we prove that $\left(S^{H}(x, y), S^{G}\left(z_{1}, z_{2}\right)\right)$ satisfies (ii). Note that, by Axiom Red, $u \in S^{H}(x, y)$ if and only if $u \in x$ or $u=y$. We consider two cases. Case 2.1. If $u \in x$, by the IH, there exists $v \in z_{1}$ such that $(u, v) \in \Phi$. Besides, by Axiom Red, $v \in S^{G}\left(z_{1}, z_{2}\right)$. Case 2.2. If $u=y$, there is $v=z_{2}$ such that $(u, v) \in \Phi$. Besides, by Axiom Red, $v \in S^{G}\left(z_{1}, z_{2}\right)$.

We now prove that $\Phi$ satisfies the following property:

(5') For every $x \in H$ and $z \in G$, if $(x, z) \in \Phi$, then (i) for every $v \in z$ there exists $u \in x$ such that $(u, v) \in \Phi$, and (ii) for every $u \in x$ exists $v \in z$ such that $(u, v) \in \Phi$.

Suppose, for the purpose of contradiction, that there exists $\left(x^{\prime}, z^{\prime}\right) \in \Phi$ such that $\left(x^{\prime}, z^{\prime}\right)$ does not satisfy (i) or does not satisfy (ii). Consider the relation $\Phi^{\prime}=\Phi \backslash\left\{\left(x^{\prime}, z^{\prime}\right)\right\}$. We now prove that $\Phi^{\prime} \subset \Phi$ is a relation which satisfies (1'), (2'), (3'), and (4'), which contradicts the fact that $\Phi$ is a least relation. We prove by weak induction on $x \in H$ that $\Phi^{\prime}$ satisfies (3') and (4').

Basis: By Axioms Zer and Ext, we prove that $0^{H}$ and $0^{G}$ have no elements. From this, we prove that $\left(0^{H}, z\right)$ satisfies (i) and (ii) if and only if $z=0^{G}$. Consider $(y, z) \in \Phi^{\prime}$. By Zer, Ext and RedSince $y$ is the only element of $S^{H}\left(0^{H}, y\right)$, and $z$ is the only element of $S^{G}\left(0^{G}, z\right)$, and since $(y, z) \in \Phi^{\prime}$, then $\left(S^{H}\left(0^{H}, y\right), S^{G}\left(0^{G}, z\right)\right) \in \Phi^{\prime}$ and it satisfies (i) and (ii).

Induction Hypothesis: Suppose that $x$ satifies (3') and (4'). 
Induction Step: Suppose $\left(x, z_{1}\right),\left(y, z_{2}\right) \in \Phi^{\prime}$. If $S^{H}(x, y) \neq x^{\prime}$ or $S^{G}\left(z_{1}, z_{2}\right) \neq z^{\prime}$, we are done, because, as shown in the proof that $\Phi$ satisfies (4'), $\left(S^{H}(x, y), S^{G}\left(z_{1}, z_{2}\right)\right)$ satisfies (i) and (ii). Thus, suppose, for the purpose of contradiction, that $S^{H}(x, y)=x^{\prime}$ and $S^{G}\left(z_{1}, z_{2}\right)=z^{\prime}$. By Axiom Red, $v \in S^{G}\left(z_{1}, z_{2}\right)$ if and only if $v \in z_{1}$ or $v=z_{2}$. We consider two cases. Case 1.1. If $v \in z_{1}$, then by the IH, there is $u \in x$ such that $(u, v) \in \Phi^{\prime}$. Besides, by Axiom Red, $u \in S^{H}(x, y)$. Case 1.2. If $v=z_{2}$, then there is $u=y$ such that $(u, z) \in \Phi^{\prime}$. Besides, by Axiom Zer, $u \in S^{H}(x, y)$. So we conclude $\left(x^{\prime}, z^{\prime}\right)$ satisfies (i). Similarly, by Axiom Red, $u \in S^{H}(x, y)$ if and only if $u \in x$ or $u=y$. We consider two cases. Case 2.1. If $u \in x$, by the IH, there is $v \in z_{1}$ such that $(u, v) \in \Phi^{\prime}$. Besides, by Axiom Red, $v \in S^{G}\left(z_{1}, z_{2}\right)$. Case 2.2. If $u=y$, there is $v=z_{2}$ such that $(u, v) \in \Phi^{\prime}$. Besides, by Axiom Red, $v \in S^{G}\left(z_{1}, z_{2}\right)$. Hence, $\left(x^{\prime}, z^{\prime}\right)$ satisfies (i) and (ii), a contradiction. Thus $\left(S^{H}(x, y), S^{G}\left(z_{1}, z_{2}\right)\right) \neq\left(x^{\prime}, z^{\prime}\right)$ and $\left(S^{H}(x, y), S^{G}\left(z_{1}, z_{2}\right)\right) \in \Phi^{\prime}$.

So we conclude that $\Phi$ satisfies ( $\left.5^{\prime}\right)$. Now we prove that $\Phi$ is a function, i.e, for all $z_{1}, z_{2} \in G$, if $\left(x, z_{1}\right),\left(x, z_{2}\right) \in \Phi$, then $z_{1}=z_{2}$. The proof goes by strong induction on $x$.

Basis: As was previously argued, $\left(0^{H}, z\right)$ satisfies (i) and (ii) if and only if $z=0^{G}$. Since $\left(0^{H}, 0^{G}\right), \Phi$ is well-defined for $x=0^{H}$.

Induction Hypothesis: Suppose the claim is true for all $y \in x$.

Inductive Step: Suppose $\left(x, z_{1}\right),\left(x, z_{2}\right) \in \Phi$. Suppose further that $w \in z_{1}$. Since $\left(x, z_{1}\right),\left(x, z_{2}\right) \in \Phi$, by $\left(4^{\prime}\right)$, there are $u, v \in G$ such that $u \in x$ and $(u, w) \in \Phi$; and $v \in z_{2}$ and $(u, v) \in \Phi$. So $(u, w) \in \Phi$ and $(u, v) \in \Phi$. Since $u \in x$, it follows from the induction hypothesis that $w=v$ and, thus, $w \in z_{2}$. Hence, for all $w \in G$, if $w \in z_{1}$, then $w \in z_{2}$ In a similar manner, for every $w \in G$, if $w \in z_{2}$, then $w \in z_{1}$. It follows from Axiom Ext that $z_{1}=z_{2}$. Now, by strong induction, it follows that $F$ is a function.

Now, we prove that $\Phi$ is injective. The proof goes by strong induction on $x_{1}$.

Basis: From above, for every $z \in G$, we have $\left(0^{H}, z\right) \in \Phi$ iff $z=0^{G}$. So the property is true for $0^{H}$.

Induction hypothesis: Suppose that the property is true for all $u \in x$.

Inductive Step: Let $y \in H$ be such that $\Phi(y)=\Phi(x)$. Let $u \in x$, that is, $S^{H}(x, u)=x$. By (3), $S^{G}(\Phi(x), \Phi(y))=\Phi\left(S^{H}(x, u)\right)=\Phi(x)=\Phi(y)$. Thus, $\Phi(u) \in \Phi(y)$. Then, by (4') from Lemma 5, there is $v \in H$ such that $v \in y$ and $\Phi(v)=\Phi(u)$. Then, by the IH, $u=v$ and, thus, $u \in y$. So we conclude that for every $u \in x$, we have $u \in y$. In a similar manner we conclude for every $u \in y$, we have $u \in x$. It follows from Axiom Ext that $x=y$.

The proof that $\Phi$ is unique is quite standard, so we omit it.

Now, we obtain, as a corollary of the Homomorphism Theorem, a version of the Categoricity Theorem (cf. Theorem 2) for the hereditary finite sets.

Theorem 6 If $\mathcal{H}=\left\langle H, 0^{H}, S^{H}\right\rangle$ and $\mathcal{G}=\left\langle G, 0^{G}, S^{G}\right\rangle$ are both HFS-models, then there exists a unique isomorphism from $\mathcal{H}$ onto $\mathcal{G}$.

Proof. Let $\left\langle H, 0^{H}, S^{H}\right\rangle$ and $\left\langle G, 0^{G}, S^{G}\right\rangle$ be two HFS-models. Consider the function $\Phi: H \rightarrow G$ given by Theorem 5. Since $\Phi$ satisfies properties (2), (3) and (4), the only condition left to prove is that $\Phi$ is surjective. The proof goes by standard induction on $z \in G$, so we omit it. 
And as a corollary of the Categoricity Theorem, we obtain the (semantical) completeness of the HFS-axioms.

Theorem 7 Let $\varphi$ be any statement in the (second order) language of the HFS-axioms. Then $\varphi$ is true on all HFS-models iff $\varphi$ is true on the hereditarily finite sets.

PROOF. By Theorem 6, any two HFS-models are isomorphic. Hence the result follows, since isomorphism preverses the truth of sentences [Awodey and Reck 2002].

Our second main result is is a version of the Converse of the Homomorphism Theorem (cf. Theorem 4) for the hereditary finite sets. First, we proof a serie of lemmas:

Lemma 1 Let $\mathcal{H}=\left\langle H, 0^{H}, S^{H}\right\rangle$ and $\mathcal{G}=\left\langle G, 0^{G}, S^{G}\right\rangle$ be HFS-structures such that Axiom Zer is true in $\mathcal{G}$, and let $\Phi: H \rightarrow G$ be an injective homomorphism. Then, Axiom Zer is true in $\mathcal{H}$.

Proof. Suppose, for a contradiction, that there are $x, y \in H$ such that $S^{H}(x, y)=$ $0^{H}$. Then, since $\Phi$ is an injective homomorphism, $0^{G}=\Phi\left(0^{H}\right)=\Phi\left(S^{H}(x, y)\right)=$ $S^{G}(\Phi(x), \Phi(y))$, contradicting $\mathcal{G}$ satisfies Axiom Zer.

Lemma 2 Let $\mathcal{H}=\left\langle H, 0^{H}, S^{H}\right\rangle$ and $\mathcal{G}=\left\langle G, 0^{G}, S^{G}\right\rangle$ be HFS-structures such that Axioms Ext and Ind are true in $\mathcal{G}$, and let $\Phi: H \rightarrow G$ be an injective homomorphism. Then, Axiom Ext is true in $\mathcal{H}$.

PROOF. Since $\Phi$ is an injective homomorphism, and Ind is true in $\mathcal{G}$, in a manner similar to the proof of Theorem 6, we have that $\Phi$ is a bijection and, hence, $\Phi$ is an isomorphism. The result follows, since isomorphism preverses the truth of sentences.

Lemma 3 Let $\mathcal{H}=\left\langle H, 0^{H}, S^{H}\right\rangle$ and $\mathcal{G}=\left\langle G, 0^{G}, S^{G}\right\rangle$ be HFS-structures such that Axiom Ind is true in $\mathcal{G}$, and let $\Phi: H \rightarrow G$ be an injective homomorphism. Then, Axiom Ind is true in $\mathcal{H}$.

PROOF. Similar to the proof of Lemma 2.

Lemma 4 Let $\left\langle H, 0^{H}, S^{H}\right\rangle$ and $\left\langle G, 0^{G}, S^{G}\right\rangle$ be HFS-structures and $\Phi: H \rightarrow G$ be an injective homomorphism. Then, for all $x, y \in H$, we have $y \in x$ iff $\Phi(y) \in \Phi(x)$.

Proof. Since $\Phi$ is an injective homomorphism, if $y \in x$, then $S^{G}(\Phi(x), \Phi(y))=$ $\Phi\left(S^{H}(x, y)\right)=\Phi(x)$. Thus, $\Phi(y) \in \Phi(x)$. For the converse, if $\Phi(y) \in \Phi(x)$, then $\Phi\left(S^{H}(x, y)\right)=S^{G}(\Phi(x), \Phi(y))=\Phi(x)$. Since $\Phi$ is injective and $\Phi\left(S^{H}(x, y)\right)=\Phi(x)$, then $S^{H}(x, y)=x$, i.e, $y \in x$.

Lemma 5 Let $\mathcal{H}=\left\langle H, 0^{H}, S^{H}\right\rangle$ and $\mathcal{G}=\left\langle G, 0^{G}, S^{G}\right\rangle$ be HFS-structures such that Axiom Red is true in $\mathcal{G}$, and let $\Phi: H \rightarrow G$ be an injective homomorphism. Then, Axiom Red is true in $\mathcal{H}$.

Proof. By Lemma 4, for all $z \in H, z \in S^{H}(x, y) \leftrightarrow \Phi(z) \in \Phi\left(S^{H}(x, y)\right)=$ $S^{G}(\Phi(x), \Phi(y))$. Now, by Axiom Red, $\Phi(z) \in S^{G}(\Phi(x), \Phi(y)) \leftrightarrow \Phi(z) \in \Phi(x) \vee$ $\Phi(z)=\Phi(y)$. We consider two cases. Case 1. By Lemma 4, $\Phi(z) \in \Phi(z) \leftrightarrow z \in x$. Case 2. Since $\Phi$ is injective, $\Phi(z)=\Phi(y) \leftrightarrow z=y$. So we conclude that, for all $z \in H$, $z \in S(x, y) \leftrightarrow[z \in x \vee z=y]$.

As a consequence of Lemmas 1, 2, 3, and 5, we have our version of the converse of the Homomorphism Theorem for the hereditary finite sets.

Theorem 8 If $\mathcal{H}=\left\langle H, 0^{H}, S^{H}\right\rangle$ is a HFS-structure and for any Zer-structure $\mathcal{G}=$ $\left\langle G, 0^{G}, S^{G}\right\rangle$, there exists a unique injective homomorphism from $\mathcal{H}$ into $\mathcal{G}$, then $\mathcal{H}$ is a HFS-model. 


\section{Perspectives}

We believe the work reported here may provide a general foundation for theories of intrinsically finite objects, i.e., finite objects constructed from finite objects, which are constructed from finite objects and so on, since these objects can be naturally modelled as hereditarily finite sets and their properties reduced to properties of hereditarily finite sets.

In particular, in a continuation of this work, we use this theory of hereditarily finite sets to prove the existence of cardinality functions in HFS-models - that is, functions that count the number of elements of an hereditarily finite set - thus providing a foundation for the study of the basic combinatorial principles. In particular, we use this framework to investigate the logical independence of these principles and intend to continue this work evaluating their combinatorial strenghts.

\section{References}

Ackermann, W. (1937). Die widerspruchsfreiheit der allgemeinen mengenlehre. Mathematische Annalen, 114(1):305-315.

Awodey, S. and Reck, E. H. (2002). Completeness and categoricity. part i: Nineteenthcentury axiomatics to twentieth-century metalogic. History and Philosophy of Logic, 23(1):1-30.

Cohen, P. J. (2008). Set theory and the continuum hypothesis. Courier Corporation.

Dedekind, R. (1888). Was sind und was sollen die zahlen? In Was sind und was sollen die Zahlen?. Stetigkeit und Irrationale Zahlen, pages 1-47. Braunschweig: Friedr. Vieweg \& Sohn, 1888.

Givant, S. and Tarski, A. (1977). Peano arithmetic and the zermelo-like theory of sets with finite ranks. Notices of the American Mathematical Society, 77:E51.

Kirby, L. (2009). Finitary set theory. Notre Dame Journal of Formal Logic, 50(3):227244.

Lawvere, F. W. (1964). An elementary theory of the category of sets. Proceedings of the National academy of Sciences of the United States of America, 52(6):1506.

Lawvere, F. W. and McLarty, C. (2005). An elementary theory of the category of sets (long version) with commentary. Reprints in Theory and Applications of Categories, $11: 1-35$.

Peano, G. (1889). Arithmetices principia: Nova methodo exposita. Fratres Bocca.

Previale, F. (1994). Induction and foundation in the theory of hereditarily finite sets. Archive for Mathematical Logic, 33(3):213-241.

Smolka, G. and Stark, K. (2016). Hereditarily finite sets in constructive type theory. In International Conference on Interactive Theorem Proving, pages 374-390. Springer.

Świerczkowski, S. (2003). Finite sets and Gödel's incompleteness theorems.

Takahashi, M.-o. (1976). A foundation of finite mathematics. Publications of the Research Institute for Mathematical Sciences, 12(3):577-708. 